\title{
Isolated Hypogonadotropic Hypogonadism
}

National Cancer Institute

\section{Source}

National Cancer Institute. Isolated Hypogonadotropic Hypogonadism. NCI Thesaurus.

Code C120145.

Hypogonadotropic hypogonadism not associated with a deficiency of other pituitary hormones. 